\title{
Role of the Coulomb interaction in the flow and the azimuthal distribution of kaons from heavy ion reactions
}

\author{
Z.S. Wang, Amand Faessler, C. Fuchs, V.S. Uma Maheswari and D. Kosov \\ Institut für Theoretische Physik der Universität Tübingen, D-72076 Tübingen, \\ Germany
}

\begin{abstract}
Coulomb final-state interaction of positive charged kaons in heavy ion reactions and its impact on the kaon transverse flow and the kaon azimuthal distribution are investigated within the framework of QMD ( Quantum Molecular Dynamics ) model. The Coulomb interaction is found to tend to draw the flow of kaons away from that of nucleons and lead to a more isotropic azimuthal distribution of kaons in the target rapidity region. The recent FOPI data have been analyzed by taking into accout both the Coulomb interaction and a kaon in-medium potential of the strong interaction. It is found that both the calculated kaon flows with only the Coulomb interaction and with both the Coulomb interaction and the strong potential agree within the error bars with the data. The kaon azimuthal distribution exhibits asymmetries of similar magnitude in both theoretical approaches. This means, the inclusion of the Coulomb potential makes it more difficult to extract information of the kaon mean field potential in nuclear matter from the kaon flow and azimuthal distribution data.
\end{abstract}

Key words: Kaons, Coulomb interaction, transverse flow, azimuthal distribution, QMD

PACS numbers: 25.75. $+\mathbf{r}$

\section{Introduction}

The importance of Coulomb final-state interaction in particle production in heavy ion reactions has been pointed out more than one decade ago [1-3]. In the energetic collision of two nuclei the Coulomb field has been shown to be able to cause a momentum shift of charged particles and distort the phase space of these particles. Although both effects are reduced to some extent due 
to the finite expansion of the charged sources, they are still very essential in understanding the observed $\pi^{-} / \pi^{+}$ratios. On the other hand, production of kaons in heavy ion reactions has attracted much attention since it has been found to be sensitive on the compressibility of nuclear matter $[4,5]$. So it is of high interest to study possible effects of final-state Coulomb interaction of charged kaons. In this work we intend to check the influence of Coulomb interaction on the transverse flow and the azimuthal distribution of positive charged kaons. Just recently, G.Q.Li, C.M.Ko and B.A.Li[6] indicated that these observables can act as an unique probe to the kaon mean field potential in dense nuclear matter. The knowlege about in-medium modifications of the properties of kaons is important not only to nuclear physics but also to astrophysics, since it is related to the possible formation of kaon condensation in the core of neutron stars [8,9]. It was mentioned that kaon Coulomb interaction has been included in the first calculation of the kaon flow by $\mathrm{Li}$ et al. based on the RBUU (Relativistic Boltzmann-Uhlenbeck-Uehling ) model [6]. However, the magnitude and the observable features of the Coulomb effect

are not presented there. Since kaon spectra are caused by a combination of different physical causes, namely by the in-medium potential of the strong interaction, by the Coulomb interaction and also the rescattering of kaons with baryons and pions and by others, we feel it necessary to make clear the role of the Coulomb final-state interaction in determining the kaon flow and the kaon azimuthal distribution. The inclusion of these effects is important to extract information about the kaon mean field potential in dense matter from the analysis of the kaon flow and azimuthal distribution.

This paper is organized as following. In section 2 we describe the methods used to treat $K^{+}$production and the Coulomb effect in heavy ion reactions. A main source for $K^{+}$production is the pion-baryon reaction leading to a kaon plus a hyperon. In section 3 we compare with the experimental data from the FOPI collaboration. In section 4 we summarize the main results of this work.

\section{Description of $K^{+}$production and the Coulomb effect}

In this work we concentrate on heavy ion reactions at SIS energies (about 1-2 $\mathrm{GeV} / \mathrm{A}$ ) since the first experiment of the kaon transverse flow has been done in this energy region by the FOPI collaboration [12]. In order to evaluate the effects of Coulomb interaction one needs a dynamic model which can describe the charge distribution in the colliding nuclei as well as the compression and expansion of the charged sources. In this work we adopt the framework of the QMD (Quantum Molecular Dynamics) model which meets these requirements. It can also distinguish between different isospin states of nucleons, $\Delta$ and $N^{*}$ resonances since an effective baryon-baryon Coulomb interaction has been implemented in QMD $[10,11]$. We have extended the model by taking 
into account pion-baryon Coulomb interaction [20]. This is of importance in providing a correct pion distribution since the pion phase space has been found to be highly isospin dependent. The charges of nucleons, nucleonic resonances (They are mainly $\Delta$ and $N^{*}$ resonances.) and pions are the source of the Coulomb field acting on $K^{+}$mesons at GSI incident energies. The degrees of freedom of these particles are treated in a non-perturbative way in our QMD model.

In our model, the $K^{+}$mesons are produced via either baryon-baryon collisions or pion-baryon annihilation processes into kaons and hyperons. For the baryonbaryon channel, we have adopted a calculated cross section based on the oneboson-exchange (OBE) model [21], rather than the parametrization given by Randrup and Ko [22], since a recent experiment has found that the latter parametrization[22] overestimates kaon production near threshold more than two orders of magnitude [19]. For the pion-baryon channel we have used a cross section calculated by using a resonance model [23]. This cross section includes the contribution from relevant resonances up to $2 \mathrm{GeV}$ and agrees well with the existing data. In our previous work [13], we have shown that the pion-induced channel has a substantial contribution to kaon production for the incident energy region of $1-2 \mathrm{GeV} / \mathrm{A}$ and for large systems. With the inclusion of this production channel we could reproduce the recent Kaos data for both the momentum spectra and the mass dependence of $K^{+}$multiplicities, however, with a realistic momentum dependent nuclear mean field. Due to the very small abundance of kaons in the energy region we have adopted the standard perturbative method to treat kaon degrees of freedom. So $K^{+}$mesons can not affect the reaction dynamics. Due to charge conservation, external Coulomb fields have no effect on the production processes of $K^{+}$mesons. After production of a kaon, it propagates in the Coulomb field generated by charged nucleons, $\Delta$ 's, $N^{*}$ resonances and pions. The Hamiltonian of kaons can be written as

$$
H_{k}=\sum_{1_{k}}\left(\sqrt{m_{k}^{2}+\vec{P}_{i_{k}}^{2}}+U_{i_{k}}^{C o u}\right)
$$

where $U_{i_{k}}^{C o u}$ is the Coulomb potential acting on kaons,

$$
U_{i_{k}}^{\text {Cou }}=\sum_{i_{k}} \frac{Z_{i_{k}} Z_{i_{n}} e^{2}}{\left|\vec{r}_{i_{k}}-\vec{r}_{i_{n}}\right|}+\sum_{i_{r}} \frac{Z_{i_{k}} Z_{i_{r}} e^{2}}{\left|\vec{r}_{i_{k}}-\vec{r}_{i_{r}}\right|}+\sum_{i_{\pi}} \frac{Z_{i_{k}} Z_{i_{\pi}} e^{2}}{\left|\vec{r}_{i_{k}}-\vec{r}_{i_{\pi}}\right|} .
$$

Here $i_{k}, i_{n}, i_{r}$ and $i_{\pi}$ denote kaons, nucleons, resonances $\left(\Delta\right.$ and $\left.N^{*}\right)$ and pions, respectively. The corresponding charges are $Z_{i_{k}}, Z_{i_{n}}, Z_{i_{r}}$ and $Z_{i_{\pi}}$. One has to include the Coulomb effects from charged nucleonic resonances and pions at the beam energies of interest, since a substantial number of nucleonic reso- 
nances are created via violent nucleon-nucleon collisions. The decay of these resonances leads to non negligible abundances of pions. However, pion abundances in heavy ion reactions are strong isospin dependent. For nuclei with high isospin asymmetry a large excess of negative charged pions compared to positive charged pions has been observed. By using the isobar model [24], the $\pi^{-} / \pi^{+}$ratio can be approximately evaluated. For instance, in the case of a reaction system as $\mathrm{Au}+\mathrm{Au}$ one expects a ratio of $\pi^{-} / \pi^{+}$as about 1.95 for pions from $\Delta$ decay, and 1.75 for pions from $N^{*}$ decay. Since the total charge of the reaction system is conserved exactly, the baryons are more positive charged during the reaction than in the ground state of the target and the projectile. Thus, it is necessary to take into account the contribution from resonances and pions in order to avoid any overestimation of the Coulomb effect. Since it has been shown that the rescattering of kaons with nucleons can affect the flow and the azimuthal distribution of kaons too, we have included $\mathrm{K}-\mathrm{N}$ rescattering in a way similar to Ref.[14].

Since the Coulomb field on $\mathrm{K}^{+}$mesons originates from charges of baryons and pions, we feel it is necessary to make sure that these particles can be described reliably by our model. In the upper and middle panels of Fig.1 we present the transverse flow, namely average particle in-plane transverse momenta as a function of the particle rapidity of nucleons and pions in the reaction of $\mathrm{Au}+\mathrm{Au}$ at an incident energy of $1 \mathrm{GeV} / \mathrm{A}$ and at an impact parameter of $\mathrm{b}=$ $5 \mathrm{fm}$. The particle rapidities have been normalized to the projectile rapidity. All through this work, we have used a soft Skyrme force (corresponding to a nuclear matter compressibility of $\mathrm{K}=200 \mathrm{MeV}$ ) combined with a momentum dependent interaction which was adjusted to the empirical nucleon-nucleon optical potential. Our results are similar to that based on RBUU by Li et al.[6] for the same reaction in both tendency and magnitude. The slight antiflow of pions compared to the nucleon flow is due to reabsorption of pions by nucleons. In the upper and middle panels of Fig.2 we show azimuthal distributions of nucleons and pions for the same reaction. The distributions have been normalized to total multiplicities of nucleons and pions, respectively. We have used a rapidity cut as $-1.3<y_{c m} / y_{\text {proj }}<-0.7$ which corresponds to the target rapidity region. In this work we place the target nucleus in the lower and the projectile nucleus in the upper hemisphere. In Ref.[7] it was found that kaon azimuthal distribution in the target (or projectile) rapidity region is more sensitive to the strong kaon potential in dense matter than in the midrapidity region. In this rapidity region Coulomb final-state interaction is also expected to have a larger effect since the target (or projectile) spectators have a much lower expansion velocity than the fireball[1]. Our results show that in the target rapidity region the nucleon azimuthal distribution is peaked at $\phi=180^{\circ}\left(\phi=0^{\circ}\right.$ is defined as the reaction plane in the direction of the scattered projectile.) corresponding to enhanced abundances of nucleons in the lower hemisphere due to the target spectators. This feature is similar to that 
found in Ref.[7]. For pions a very weak enhancement in the upper hemisphere can be seen due to the weak antiflow of pions. In the lower panels of Fig.1 and Fig. 2 the kaon flow and the normalized kaon azimuthal distribution for the same reaction is shown. The azimuthal distribution is given for the same target rapidity region. Both results with and without kaon Coulomb interaction are shown. The flow and azimuthal distribution obtained in the case of no Coulomb interaction follow that of the nucleons, since kaons are produced just from violent collision processes in which a nucleon or nucleonic resonance is involved. We observed a kaon flow in the same direction as that of nucleons. The kaon azimuthal distribution in the target rapidity region exhibits also an enhancement in the lower hemisphere, similar to that of nucleons. However, the final-state Coulomb interaction distorts the flow and azimuthal distribution of $K^{+}$mesons by a non trivial magnitude. It can be found from the figures that the Coulomb interaction moves the flow of $K^{+}$mesons away from that of nucleons. The preferential emission of target rapidity kaons in the lower hemisphere is suppressed by the Coulomb interaction on $K^{+}$mesons. It results in a nearly isotropic azimuthal distribution of kaons in the target rapidity region. In a quantitative language, the Coulomb interaction has changed the kaon flow parameter, which is defined as $\mathrm{F}=\left.\frac{d<P_{x}>}{d y_{c . m .}}\right|_{y_{c . m .}=0}$, from $66 \mathrm{MeV} / \mathrm{c}$ to 15 $\mathrm{MeV} / \mathrm{c}$, approximately. Since we have used an azimuthal distribution which is normalized to the total kaon multiplicity, the quantity $\Delta\left(\frac{d N}{d \phi}\right)=\left(\frac{d N}{d \phi}\right)_{\max }$ - $\left(\frac{d N}{d \phi}\right)_{\min }$ can act as a measure of the asymmetry of the distribution. The asymmetry factor of the kaon azimuthal distribution decreases from about 0.075 to 0.005 . The influence of the Coulomb interaction can be understood since positive charged kaons experience a net repulsive Coulomb field in the reaction system.

\section{Comparision with data}

Now we compare to the experimental data for central collisions of $\mathrm{Ni}+\mathrm{Ni}$ at an incident energy of $1.93 \mathrm{GeV} / \mathrm{A}$ obtained by the FOPI Collaboration[12]. We are interested to check how much freedom is left for the strong kaon potential after the inclusion of the Coulomb effect since it also plays a role in determining the kaon flow and the kaon azimuthal distribution. In order to show the contribution from the Coulomb interaction compared to that of the strong kaon in-medium potential, we have included the propagation of kaons in the strong mean field potential in our QMD model. This is also necessary if one expects agreement with the data since the strong kaon potential has been shown to contribute substantially to the kaon flow and the kaon azimuthal distribution. The Hamiltonian of kaons has the form

$$
H_{k}=\sum_{1_{k}}\left(\sqrt{m_{k}^{2}+\vec{P}_{i_{k}}^{2}}+U_{i_{k}}^{\text {Cou }}+U_{i_{k}}^{S t r}\right),
$$


where $U_{i_{k}}^{S t r}$ is the strong interaction potential acting on kaons. The longestablished formalism for studying kaon properties in dense matter is chiral perturbation theory, which treats the chiral symmetry breaking term in a chiral Lagrangian by current quark masses as a perturbation since they are very small compared to the nucleon mass. In this work we have adopted a potential suggested by Brown and Rho[25]. It includes not only the interaction due to vector meson exchange and the scalar interaction originating from the symmetry breaking, but also the scalar interaction arising from virtual pair corrections. This potential takes into account also the change of the pion decay constant in the medium, where it is smaller than in free space due to a decrease of the quark condensate in nuclear matter. This potential agrees well to that from the empirical impulse approximation, and can account for the large attraction for negative charged kaons found in kaonic atoms. Some details of this potential can be found in a recent review given by Brown and Rho[25]. Since $U_{i_{k}}^{S t r}$ depends not only on the momentum of the kaon and the surrounding baryon density $\rho_{B}$, but also on the scalar density $\rho_{S}$, we have followed a method used in the RBUU model[17] to evaluate the scalar density $\rho_{S}$ as a function of the baryon density $\rho_{B}$. In this work, we have not included the effect of the strong interaction potential on the kaon production threshold in elementary collision processes, since there exist quite different opinions about this point in the literature[15,16,25,26]. With respect to enhanced pion abundances at the higher incident energies we have included the rescattering of kaons by pions as in Ref.[18]. On the other hand, kaon-nucleon rescattering is treated in a non-perturbative way as it was found to be necessary for avoiding possible violation of energy conservation[6]. In our calculation, we have used an impact parameter cut of $\mathrm{b}<4 \mathrm{fm}$, corresponding to the chosen centrality in the experiment. A $P_{t}$ cut as $P_{t} / \mathrm{m}<0.5$ has been used in agreement with the experimental acceptance. In order to make sure that our QMD model is reliable at this higher incident energy we compare also the calculated proton flow to the data in the upper panel of Fig.3. A good agreement between the calculation and the FOPI data is found. In the lower panel of Fig.3 the calculated $K^{+}$flow is shown. We have made calculations for three cases: (1) with neither Coulomb interaction nor the strong $K^{+}$potential, (2) with only the Coulomb interaction, (3) with both the Coulomb interaction and the in-medium strong kaon potential. Although for this light system the Coulomb interaction has weaker effect compared to the $\mathrm{Au}+\mathrm{Au}$ system, its contribution to the kaon flow is visible. They draw the calculated kaon flow closer to the data. It can be seen that the result with only the Coulomb interaction lies already whithin the error bars of the data. On the other hand, the result with both the Coulomb interaction and the strong interaction potential agrees also with the data. It is interesting to note that if our calculations differ only in the kaon potential of the strong interaction, both resultant flows for the kaons of cases (2) and (3) from above are whithin the error bars of the data. It means that the experimental precision is not enough to see clearly the change in the kaon flow caused by the in-medium potential used here. In Fig.4 we 
present the calculated kaon azimuthal distribution for the same three cases as in Fig.3. Here we have adopted the same $P_{t}$ cut. A rapidity cut of $-1.2<$ $y_{c m} / y_{\text {proj }}<-0.8$ corresponding to the target rapidity region has been used in getting the distributions. It can be found from Fig.3 and Fig.4 that the change in the kaon flow and azimuthal distribution caused by the Coulomb final-state interaction has the same tendency as caused by an in-medium strong kaon potential. This can be undersood since both the Coulomb interaction and the strong interaction potential are effectively repulsive for $K^{+}$mesons. We note that the preliminary data from the FOPI collaboration[12] show no significant asymmetry in the kaon azimuthal distribution in the target rapidity region. With neither a Coulomb nor a strong interaction potential the distribution has a peak around the lower hemisphere, and the corresponding value of asymmetry factor $\Delta\left(\frac{d N}{d \phi}\right)$ is about 0.046 . The Coulomb interaction decreases the peak and leads to a smaller value of $\Delta\left(\frac{d N}{d \phi}\right)$ as 0.032 . The strong potential suppresses further the emission of kaons in the lower hemisphere and changes the peak to a dip. However, the resultant asymmetry factor $\Delta\left(\frac{d N}{d \phi}\right)$ is about 0.027, which is very similar to that obtained with only the Coulomb interaction. These results concerning the flow and the azimuthal distribution of kaons mean that after taking into account the Coulomb interaction it becomes more difficult to extract information about the strong kaon potential by analyzing the data of the kaon flow and the kaon azimuthal distribution. Experimental data with higher precision are needed.

\section{Summary}

In summary, we have invesigated the effect of the Coulomb interaction of $\mathrm{K}^{+}$ mesons with protons, charged resonances and pions on the kaon flow and the kaon azimuthal distribution. It is found that the final-state Coulomb interaction can distort the kaon flow and the kaon azimuthal distribution by a non trivial magnitude. The repulsive Coulomb interaction tends to draw the flow of kaons away from that of nucleons and leads to a more isotropic azimuthal distribution of kaons in the target rapidity region. These effects are similar in tendency to that caused by the strong kaon in-medium potential. The data obtained recently by the FOPI collaboration have been analyzed with both the Coulomb interaction and a kaon in-medium potential of the strong interaction included. It is found that both the calculated kaon flow with only the Coulomb interaction and with both the Coulomb interaction and the strong $K^{+}$potential lie within the error bars of the data. The kaon azimuthal distribution in the same two cases exhibits an asymmetry of very similar magnitude, although the peaks in the two distributions have different positions. It means, after the Coulomb effect is taken into account, it becomes more difficult to extract information of the kaon mean field potential in nuclear matter from the 
kaon flow and the kaon azimuthal distribution. Higher precision of the experimental data is needed to draw a reliable conclusion on the $K^{+}$strong potential. 


\section{References}

[1] W.Benenson et al., Phys.Rev.Lett. 43 (1979) 683; K.G.Libbrecht and S.E.Koonin, Phys.rev.Lett. 43 (1979) 1581; G.Bertsch, Nature 283 (1980) 280; M.Gyulassy and S.K.Kauffmann, Nucl.Phys.A 362 (1981) 503.

[2] S.A.Bass, C.Hartnack, H.Stöcker and W.Greiner, Phys.Rev.C51 (1994) 3343.

[3] T.Osada, S.Sano, M.Biyajima and G.Wilk, Phys.Rev.C54 (1996) R2167.

[4] J.Aichelin and C.M.Ko, Phy.Rev.Lett.55 (1985) 2661.

[5] S.W.Huang, Amand Faessler, G.Q.Li, R.K.Puri, E.Lehmann, D.T.Khoa and M.A.Matin, Phys.Lett.B298 (1993) 41.

[6] G.Q.Li, C.M.Ko and Bao-An Li, Phys.Rev.Lett.74 (1995) 235. G.Q.Li and C.M.Ko, Nucl.Phys.A594 (1995) 460.

[7] G.Q.Li, C.M.Ko and G.E.Brown, Phys.Lett.B381 (1996) 17.

[8] D.B. Kaplan and A.E. Nelson, Phys.Lett.B173 (1986) 57.

[9] G.E.Brown and H.A.Bethe, Astrophys. J.423 (1994) 659.

[10] J.Aichelin and H.Stöcker, Phys.Lett.B176 (1986) 14.

[11] J.Aichelin, Phy.Reports.202 (1991) 233.

[12] J.L.Ritman and the FOPI Collaboration, Z.Phys.A352 (1995) 355.

[13] C.Fuchs, Z.S.Wang, L.Sehn, Amand Faessler, V.S. Uma Maheswari and D. Kosov, Phys.Rev.C, to be published.

[14] X.S.Fang, C.M.Ko and Y.M.Zheng, Nucl.Phys.A556 (1993) 499.

[15] X.S.Fang, C.M.Ko, G.Q.Li and Y.M.Zheng, Nucl.Phys.A575 (1994) 766.

[16] W.Cassing, E.L.Bratkovskaya, U.Mosel, S.Teis and A.Sibirtsev, Phys.Rev.C, in press.

[17] K.Weber, B.Blättel, W.Cassing, H.-C. Dönges, V.Koch, A.Lang and U.Mosel, Nucl.Phys.A 539 (1992) 713.

[18] C.M.Ko, Phys.Rev.23 (1981) 2760.

[19] J.T.Balewski and COSY-11 collaboration, Phys.Lett.B, to be published.

[20] V.S.Uma Maheswari, C.Fuchs, Amand Faessler, L.Sehn, D.Kosov and Z.S.Wang, submitted to Nucl.Phys.A.

[21] A.Sibirtsev, Phy.lett.B359 (1995) 29.

[22] J.Randrup and C.M.Ko, Nucl.Phys.A343 (1980)519. 
[23] K.Tsushima, S.W.Huang and Amand Faessler, Phys.Lett.B337 (1994) 245; K.Tsushima, S.W.Huang and Amand Faessler, J.Phys.G21 (1995) 33.

[24] R.Stock, Phys.Reports.135 (1986) 259.

[25] G.E.Brown and Mannque Rho, Phys.Reports.269 (1996) 333, and references there.

[26] W.Weise, Nucl.Phys.A553 (1993) 59c. 


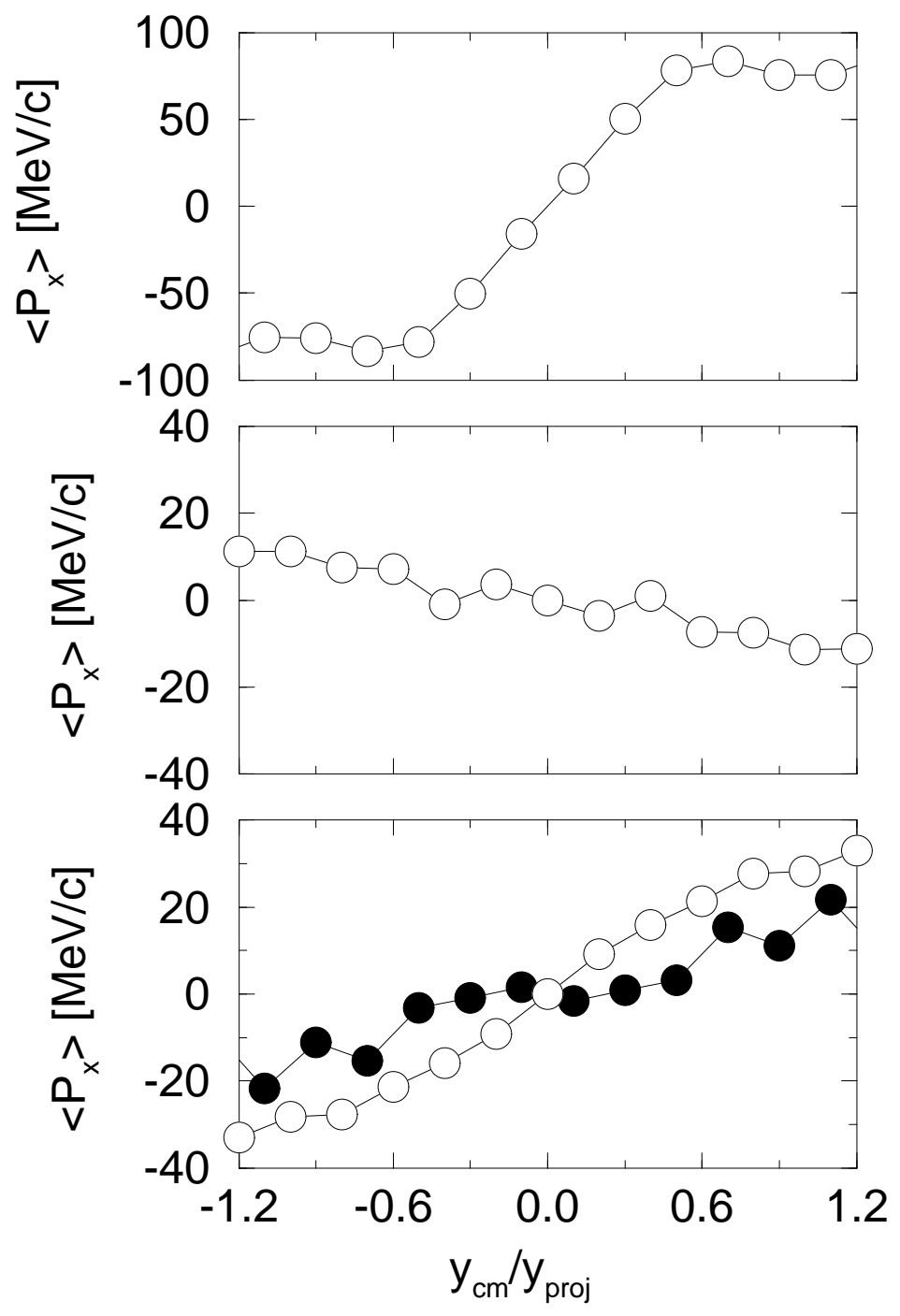

Fig. 1. Transverse flows of nucleons (upper panel) and pions (middle panel) as well as positive charged kaons (lower panel) in the reaction $\mathrm{Au}+\mathrm{Au}$ at incident energy $\mathrm{E} / \mathrm{A}=1 \mathrm{GeV}$ and at impact parameter $\mathrm{b}=5 \mathrm{fm}$. In the lower panel, the open circles denote the calculated kaon flow without any final-state Coulomb interaction, while the filled circles correspond to the result with the kaon Coulomb interaction. 

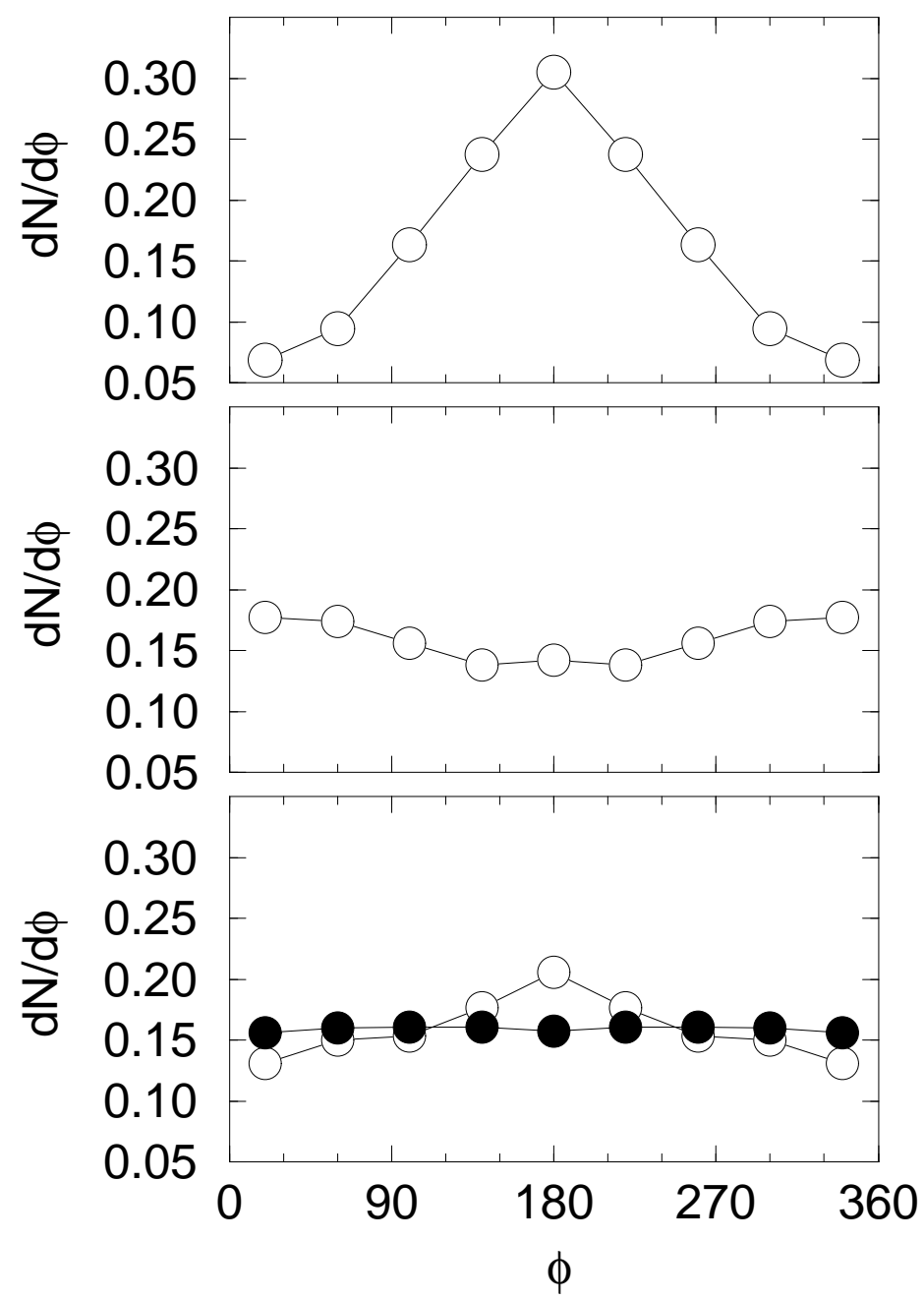

Fig. 2. Normalized azimuthal distributions of nucleons (upper panel) and pions (middle panel) as well as positive charged kaons (lower panel) in the reaction of $\mathrm{Au}+\mathrm{Au}$ at incident energy $\mathrm{E} / \mathrm{A}=1 \mathrm{GeV}$ and at impact parameter $\mathrm{b}=5 \mathrm{fm}$. A rapidity cut of $-1.3<y_{c m} / y_{\text {proj }}<-0.7$ which corresponds to the target rapidity region has been used in getting the distributions. In the lower panel, the open circles denote the calculated kaon azimuthal distribution without any final-state Coulomb interaction, while the filled circles correspond to the result with the kaon Coulomb interaction. 

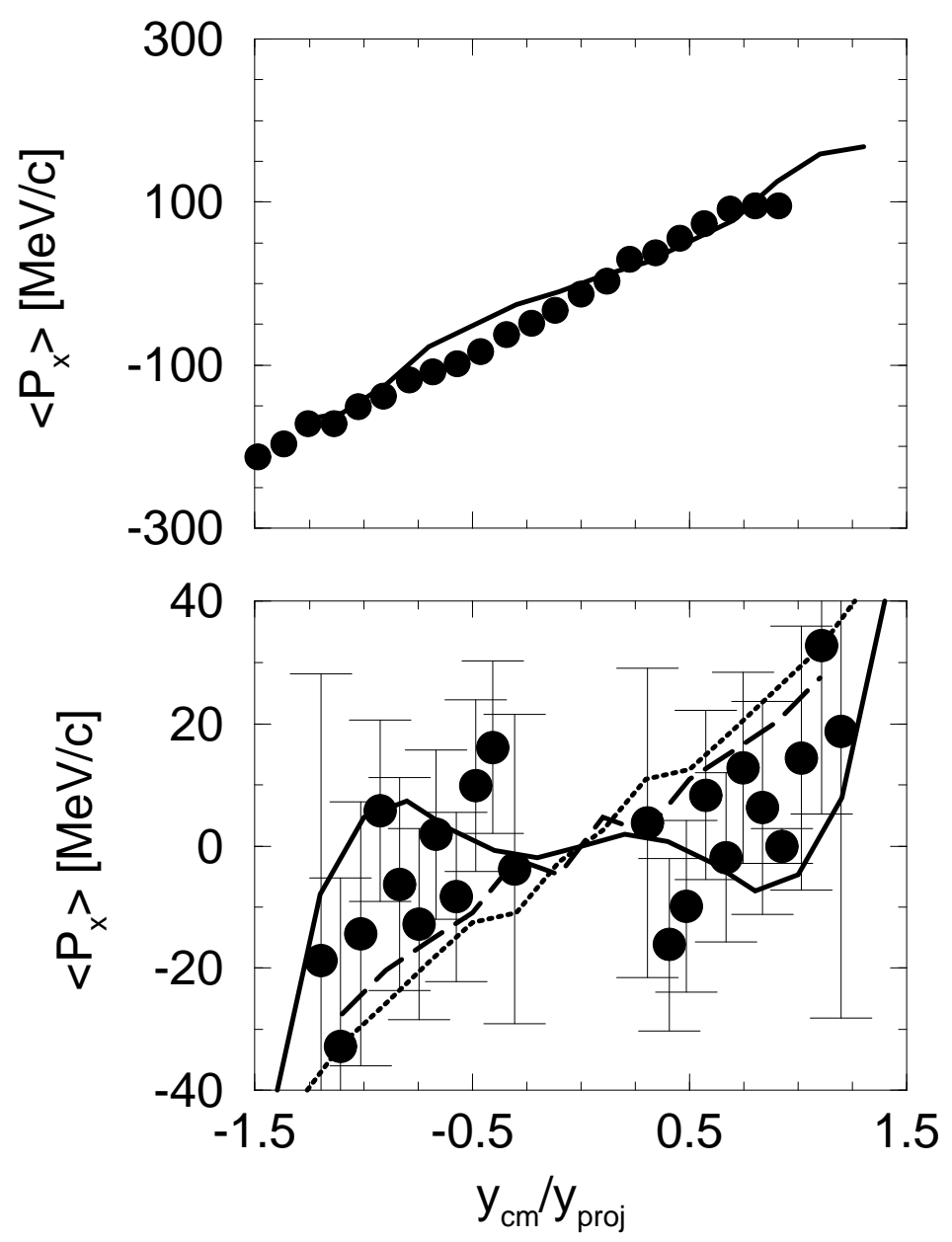

Fig. 3. Transverse flows of protons (upper panel) and positive charged kaons (lower panel) in central collisions of $\mathrm{Ni}+\mathrm{Ni}$ at incident energy $\mathrm{E} / \mathrm{A}=1.93 \mathrm{GeV}$. The circles are experimental data obtained by the FOPI group[12]. The lines are our results based on the QMD model with an impact parameter cut as $\mathrm{b}<4 \mathrm{fm}$. In the lower panel, the dotted line denotes the calculated kaon flow with neither a Coulomb nor a strong interaction potential, while the dashed line denotes the result with the Coulomb final-state interaction. The solid line corresponds to the result with both the Coulomb interaction and a potential of the strong interaction. A $P_{t}$ cut as $P_{t} / \mathrm{m}<0.5$ has been used in getting these results. 


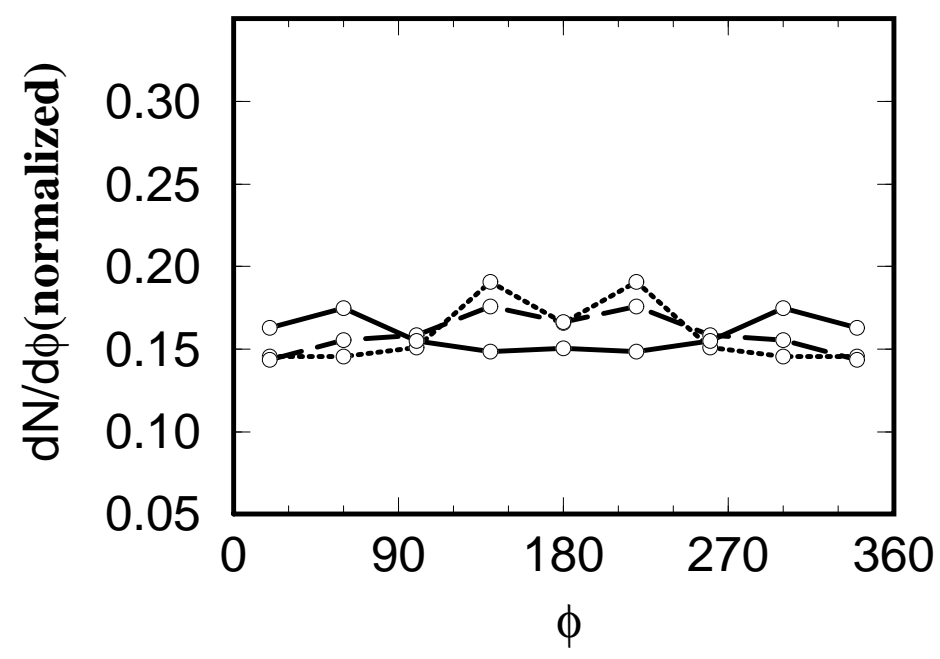

Fig. 4. Calculated normalized azimuthal distributions of positive charged kaons in central collisions of $\mathrm{Ni}+\mathrm{Ni}$ at incident energy $\mathrm{E} / \mathrm{A}=1.93 \mathrm{GeV}$. These results have been obtained based on the QMD model with an impact parameter cut as $b<4 \mathrm{fm}$. The dotted line denotes the result with neither Coulomb interaction nor a strong kaon potential, while the dashed line denotes the result with the Coulomb final-state interaction. The solid line corresponds to the result with both the Coulomb interaction and a strong kaon potential. We have used a $P_{t}$ cut as $P_{t} / \mathrm{m}<0.5$ as in the experiment. A rapidity cut of $-1.2<y_{c m} / y_{\text {proj }}<-0.8$ which corresponds to the target rapidity region has been used in getting the distributions. 